\title{
Comprehensive assessment of RegCM4 towards interannual variability of Indian Summer Monsoon using multi-year simulations
}

\author{
Suman Maity ${ }^{1}$, Sridhara Nayak ${ }^{2}$, Haraprasad Nayak ${ }^{3}$, and R Bhatla ${ }^{4}$ \\ ${ }^{1}$ Sun Yat-Sen University \\ ${ }^{2}$ Kyoto University \\ ${ }^{3}$ Indian Institute of Technology Bhubaneswar \\ ${ }^{4}$ Banaras Hindu University
}

February 3, 2022

\begin{abstract}
In this study, the Interannual variability (IAV) of Indian Summer Monsoon (ISM) is investigated using multi-year (19822016) seasonal scale simulations (May-September) of the regional climate model RegCM4 developed by International Center for Theoretical Physics, Italy. Model simulated fields such as surface temperature, wind and rainfall are validated initially to testify the climatological behaviour of ISM. Subsequently, different aspects of IAV associated with ISM are discussed primarily focusing on model simulated rainfall and are verified against high resolution rainfall analysis from India Meteorological Department (IMD). Empirical Orthogonal Function (EOF) analysis technique is also applied to identify the leading modes of IAV. Analysis indicated that RegCM4 shows reasonable accuracy in simulating major large scale features, however, has cold bias over entire India and wet (dry) bias over northwest and peninsular (central) India. Easterly (westerly) bias is noticed in the model simulated low (upper) level wind that affects regional Hadley circulation. The model bias is found to be associated with the feedback cycle of land-atmosphere interaction. Surface evaporative cooling likely affects the instability in the atmospheric column, thereby limiting the convection and thus reducing rainfall. While categorizing, it is noticed that the deficit, normal and excess rainfall years in the model simulation agrees well with the IMD observation for about half of the study period, however, the normal years are relatively better reproduced by the model than the extreme years (deficit and excess). EOF analysis revealed that first two leading modes of IMD rainfall are linked with large scale variabilities viz., El-Nino southern oscillation and Indian ocean dipole respectively but RegCM4 could not well reproduce these relationships. Eventhough, the model showed spectral peaks for 2-7 years periodicity, these peaks are very close to the red noise spectrum due to their weak power which indicated the model's limitation in capturing large scale variability. Overall, this study suggests that the RegCM4 could capture the climatological features of ISM fairly well, but needs further improvement in representing the IAV more accurately.
\end{abstract}

Note: This manuscript is published in Theoretical and Applied Climatology. https://doi.org/10.1007/s00704-022-03961-5 